\title{
Exotic Hybrid Meson Spectroscopy with the GlueX detector at JLab
}

\author{
David Lawrence ${ }^{1, a}$ and the GlueX Collaboration \\ ${ }^{1}$ Thomas Jefferson National Accelerator Facility
}

\begin{abstract}
The GlueX experiment[1] is scheduled to begin taking data in 2015. The goal is to discover evidence for the existence of exotic hybrid mesons and to map out their spectrum in the light quark sector. Recent theoretical developments using Lattice QCD [2] predict exotic hybrid states in a mass range accessible using the newly upgraded $12 \mathrm{GeV}$ electron accelerator at Jefferson Lab. Hybrid mesons, and in particular exotic hybrid mesons, provide the ideal laboratory for testing QCD in the confinement regime since these mesons explicitly manifest the gluonic degrees of freedom. The experiment will use $9 \mathrm{GeV}$ linearly polarized photons produced via coherent Bremsstrahlung to produce the exotic hybrids. The decay products will be detected in the solenoid-based GlueX detector currently under construction at Jefferson Lab. The status of the GlueX experiment including detector parameters will be presented along with theoretical motivation for the experiment.
\end{abstract}

\section{Introduction and Motivation}

Recent Lattice QCD calculations have predicted the existence of meson states in which the quark and anti-quark are bound together by a gluonic field that itself contributes to the quantum numbers of the state[2,3]. These so-called hybrid states are predicted to exist in a mass range starting at about $2 \mathrm{GeV}$ as shown in figure 1 (blue frames). The states are grouped by $J^{P C}$ quantum numbers where $J$ is the total angular momentum, $P=(-1)^{L}$ is the parity, and $C=(-1)^{L+S}$ the charge conjugation of the state. According to the constituent quark model, only certain $J^{P C}$ states are obtainable from a simple quark, anti-quark $(q \bar{q})$ pair. The "glue" however can contribute to the overall $J^{P C}$ state of the meson $(q \bar{q} g)$ allowing for the so called "exotic" states such as $0^{+-}, 1^{-+}$, and $2^{+-}$. The lightest of these is predicted to be the $1^{-+}$state as shown in the figure. A recent review of the current experimental landscape of these states can found in reference [4].

The GlueX experiment will attempt to confirm the existence of these exotic hybrid states using a new detector currently under construction at Jefferson Lab. GlueX is part of a larger project to upgrade the Jefferson Lab electron accelerator that will double its energy to $12 \mathrm{GeV}$.

\section{Photo-production and Amplitude Analysis}

The GlueX experiment will use real photons incident on protons to produce the exotic hybrid mesons. Based on LQCD calculations of radiative decay widths of charmed mesons and their agreement with

\footnotetext{
ae-mail: davidl@jlab.org
} 


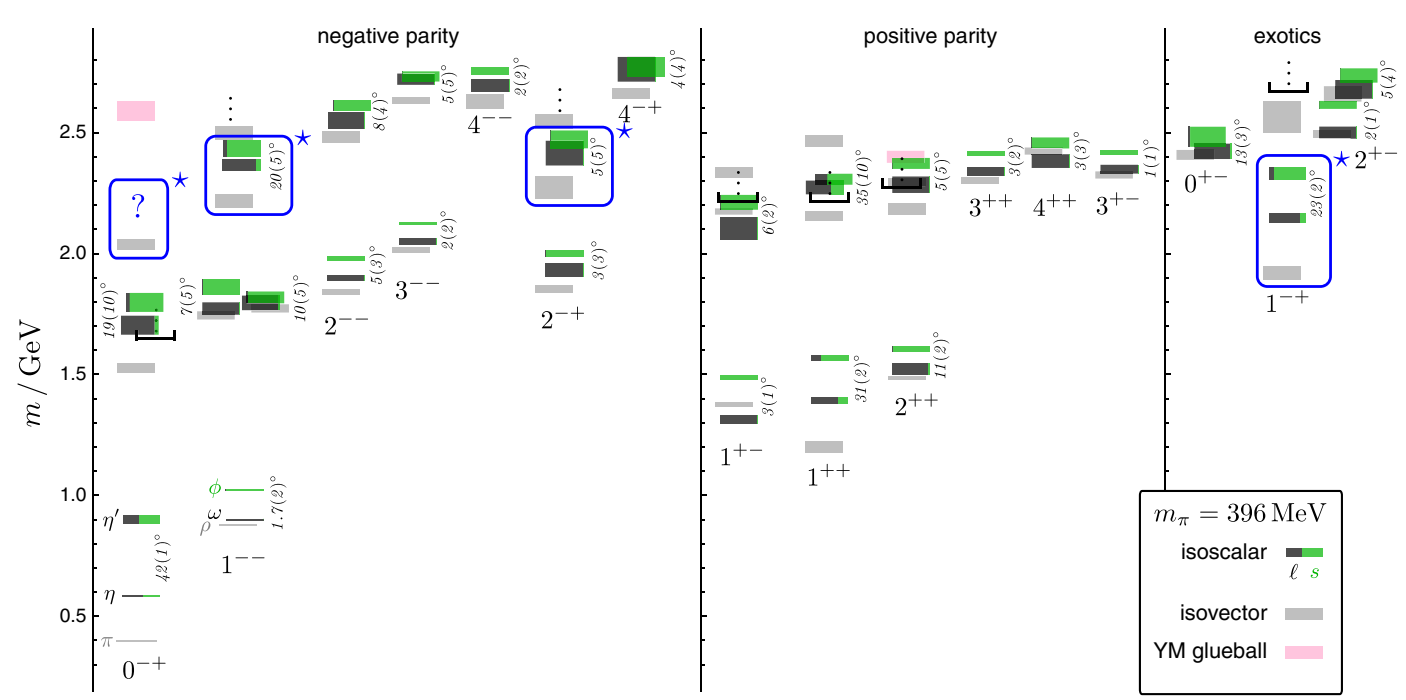

Figure 1. From ref. [2] (see figure 6). Lattice QCD calculation of isoscalar(black,green) and isovector(grey) meson spectrum using $m_{\pi} \sim 400 \mathrm{MeV}$. The black and green boxes represent the light quark $(\ell=u$ and $d)$ and the grey boxes represent the strange quark $(s)$ content of the isoscalar states. Pink boxes are glueball states arising from quarkless Yang-Mills theory[5]. The lightest hybrid meson states are indicated by the blue frames with the exotics grouped on the right hand side of the plot. Mixing angles between the light quark $(\ell)$ and strange quark $(s)$ parts are also shown in degrees.

experiment [6], it is expected that photo-production of light quark sector exotic hybrids will be similar in strength with non-exotic mesons. The spin-1 component of the photon wave function allows an exotic hybrid to be generated without incurring an additional spin flip transition. Contrast this with the case for charged pion probes where the spins of the constituent quarks are anti-aligned. The linear polarization will also give an indication on the naturality of the exchanged particle in the reaction.

The mass region where the exotic hybrids are predicted $(\sim 2 \mathrm{GeV})$ is occupied by numerous overlapping non-exotic states. Identifying these states via simple "peak hunting" is not possible. Instead, an amplitude (or partial wave) analysis must be done to identify contributions of each of the states at a given invariant mass. Figure 2 shows a Monte Carlo exercise that helps illustrate the technique. For this exercise, the $3 \pi$ invariant mass spectrum is generated using amplitudes for 5 states, one of which being the exotic $\pi_{1}(1600)$ (see ref. [4] and references therein). The $\pi_{1}(1600)$ was generated such that it contributed $1.6 \%$ of the total cross section. Likelihoods are formed using all events in the sample and a set of multipliers for the amplitudes. The likelihood is maximized to determine the optimal set of multipliers at each invariant mass bin. The bottom plot shows the exotic wave multiplier as a function of invariant mass, clearly indicating a peak at the generated mass of $1.6 \mathrm{GeV}$. The width and relative phase of the states are also obtained from the analysis and show the experiment is sensitive to signals that make up less than a percent of the total rate.

\section{The GlueX detector}

Figure 3 shows a drawing of the GlueX detector and photon tagger. The distance between the tagger and the GlueX detector is not drawn to scale. Details on the photon tagger are given in section 3.1. The GlueX detector is designed for a fixed target experiment using a superconducting solenoidal magnet. 
Figure 2. Amplitude analysis exercise using Monte Carlo data. Amplitude analysis is a necessary tool for identifying quantum states that are overlapping in phase-space with other, more abundantly produced states. In this exercise, the $\pi_{1}(1600)$ state shown by the red markers in the $3 \pi$ invariant mass spectrum in the top plot is extracted using an Amplitude Analysis to obtain the bottom plot where the yield (red points) and relative phase (black points) are shown.

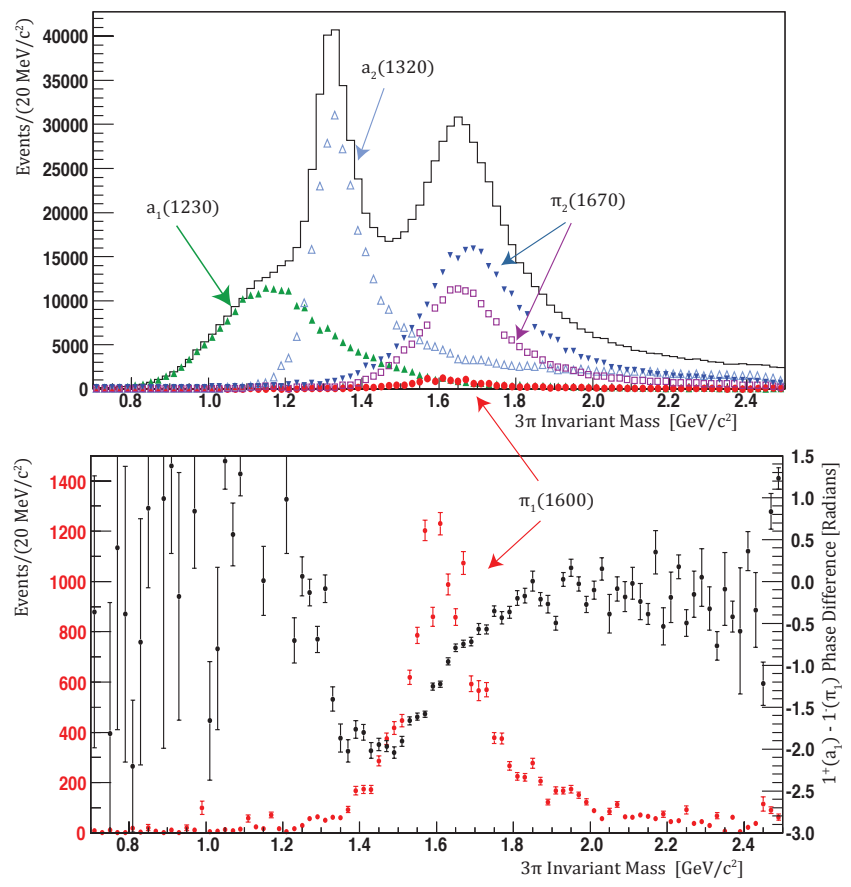

The interior of the magnet and downstream regions are outfitted with detectors to provide very high acceptance while maintaining good detector resolution. Details on some of the individual detector systems that make up the GlueX detector are given in sections 3.2-3.3.

\subsection{Linearly polarized Photon Source}

The GlueX experiment will use a linearly polarized photon beam created using a coherent Bremsstrahlung technique in which $12 \mathrm{GeV}$ electrons from the CEBAF accelerator are passed through a thin $(\sim 20 \mu \mathrm{m})$ diamond radiator. Some small fraction of the electrons will generate a photon via Bremsstrahlung (either coherently or incoherently). Photons that are produced coherently will be linearly polarized and concentrated in narrow energy regions called coherent peaks. The location of these peaks in the energy spectrum can be tuned by adjusting the angle of the diamond with respect to the electron beam direction. The coherently produced (polarized) photons will also have a higher concentration at forward angles than incoherently produced (unpolarized) photons. In order to increase the fraction of polarized to unpolarized photons hitting the GlueX target, a collimator is placed approximately $75 \mathrm{~m}$ downstream of the diamond radiator. Figure 4 shows the energy spectrum of photons that will be produced at the radiator and those that will make it to the liquid Hydrogen target. The coherent energy peak shown in the figure can be tuned by adjusting the angle of the diamond lattice with respect to the electron beam direction. For GlueX, this will be tuned such that the peak lies between $8.4 \mathrm{GeV}$ and $9.0 \mathrm{GeV}$. For this energy setting, the polarization at the peak will be $40 \%$.

The photon tagger measures the energy and time of photons in the beam. The electrons that undergo Bremsstrahlung will be bent away from the primary electron beam into a detector, thereby measuring their residual momentum. The tagger consists of a long dipole magnet with a $1.5 \mathrm{~T}$ field. The area corresponding to the photon energy coherent peak at $8.4 \mathrm{GeV}-9.0 \mathrm{GeV}$ is covered by scintillation detectors with a fine segmentation providing a photon energy resolution of $\sim 0.1 \%$. 


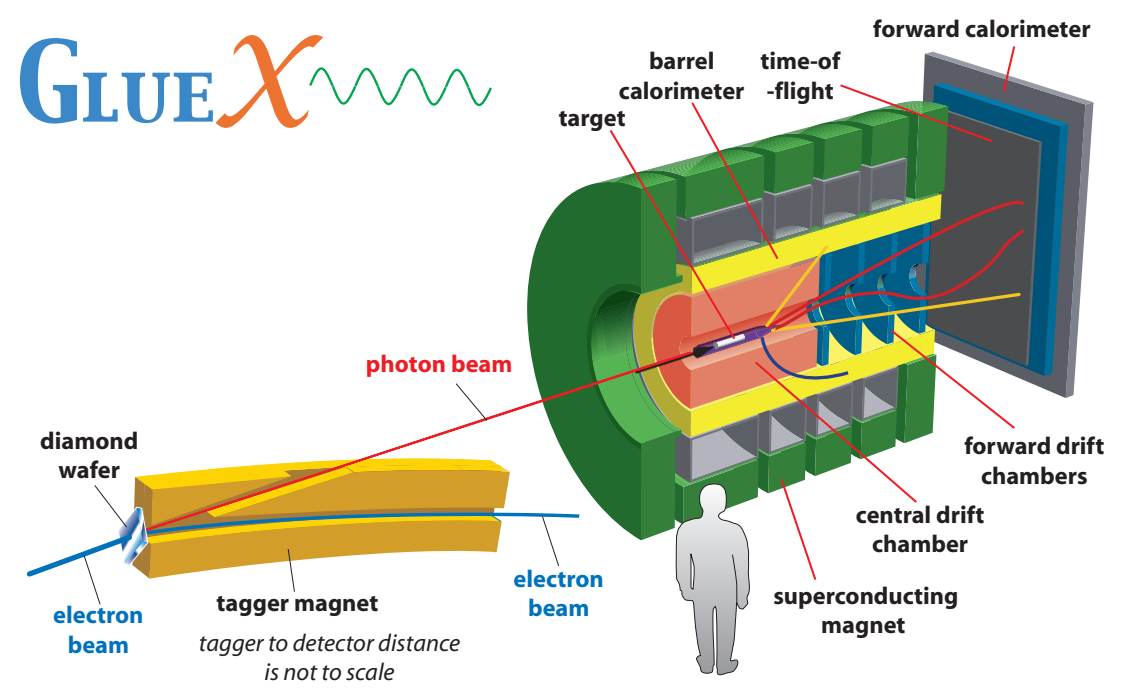

Figure 3. A rendering of the GlueX detector and photon tagger. The distance shown between the tagger magnet and superconducting magnet is not drawn to scale. See text for details on each of the detector systems.

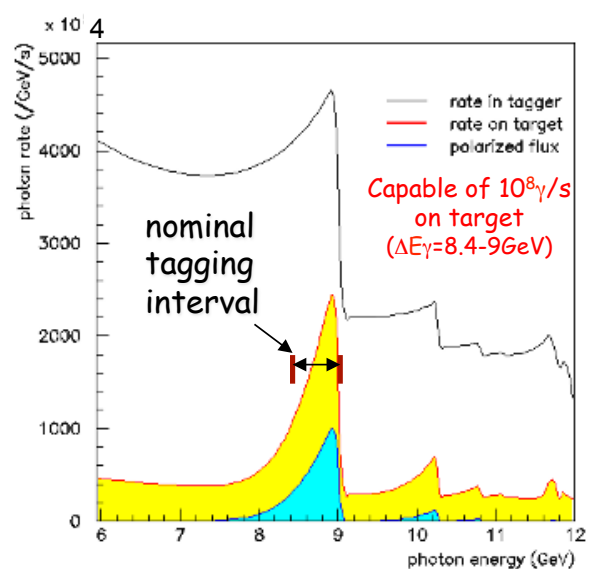

Figure 4. Energy spectrum of photons generated by the photon source. The black curve represents the total spectrum of both coherent and incoherent photons produced in the diamond radiator. The yellow spectrum is what is left after passing the beam through the collimator $\sim 80 \mathrm{~m}$ downstream. The cyan color represents the polarized part of the yellow spectrum. A 1.5T dipole magnet will be used to bend the post-Bremsstrahlung electrons into a detector plane so that the photon energy may be tagged via the residual electron momentum. The high resolution tagging range is indicated in the figure as "nominal tagging interval".

\subsection{Charged Particle Tracking}

Charged particle tracking in GlueX will be done using two drift chambers. Figure 5 shows the Central Drift Chamber (CDC), which will be installed in the upstream region of the superconducting solenoid. The CDC straw tubes have a position resolution of $\sim 150 \mu \mathrm{m}$. Figure 6 shows the Forward Drift Chamber FDC, which will be installed in the downstream region of the solenoid. It will provide 3D space points with $\sim 200 \mu \mathrm{m}$ resolution in the coordinates perpendicular to the beam line. The CDC and FDC together provide coverage in the range $1^{\circ}<\theta<160^{\circ}$ with some particles traversing parts of both detectors. Overall momentum resolution is $\sigma_{p} / p \approx 1-3 \%$ for tracks with $\theta>5^{\circ}$. 
INPC 2013

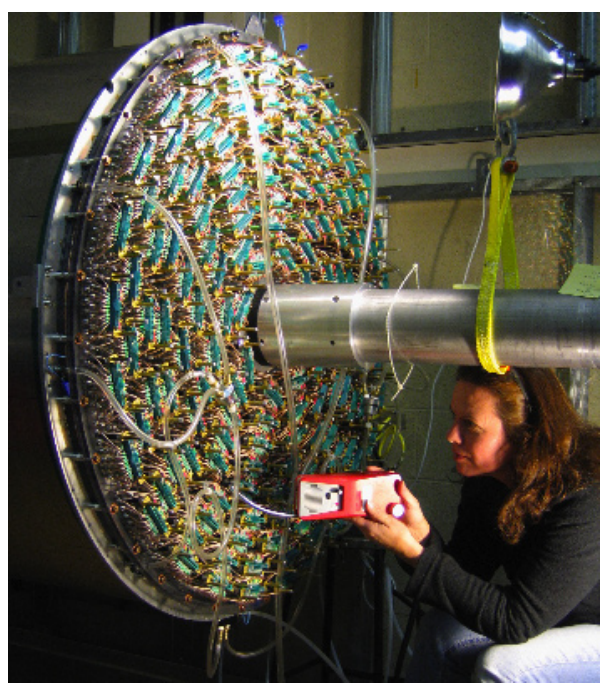

Figure 5. Central Drift Chamber (CDC) being tested for gas leaks. The CDC is a straw tube drift chamber with approximately 3500 straw tubes. It is made from alternating sections of axial (parallel to the beam direction) and stereo ( $6^{\circ}$ angle with respect to the beam direction). This allows the track's polar angle $(\theta)$ to be determined when multiple layers are considered. The end plate shown is populated with the amplifier electronics which instrument the wires to the left.

Figure 6. The GlueX Forward Drift Chamber (FDC) assembled and ready for final testing. The FDC consists of four separate packages, each with six chambers. Each chamber provides signals both from the wires and the cathode strips. Both the upstream and downstream cathode planes are instrumented for readout. This provides information on the position along the hit wire improving the pattern recognition.

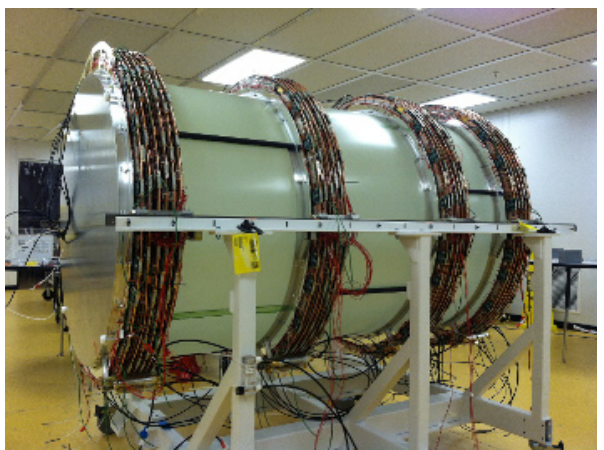

\subsection{Calorimetry}

The GlueX detector contains two electromagnetic calorimeters shown in figures 7 and 8 . The Barrel Calorimeter (BCAL) lines the inside bore of the superconducting solenoid. It will detect photons emerging from the target with $\theta \gtrsim 12^{\circ}$ and $E_{\gamma}>60 \mathrm{MeV}$. The Forward Calorimeter (FCAL) is downstream of the magnet (approximately $6 \mathrm{~m}$ downstream of the target). It will detect photons in the forward direction between $2^{\circ}$ and $11^{\circ}$, with $E_{\gamma} \gtrsim 150 \mathrm{MeV}$. The BCAL has a photon energy resolution of $(5.54 / \sqrt{E} \oplus 2.0) \%$ and with double ended readout is able to measure the photon position along the beam direction with a resolution of $\sigma_{z} \sim 0.5 \mathrm{~cm} / \sqrt{E}$. The FCAL has an energy resolution of $(5.7 / \sqrt{E} \oplus 1.6) \%$ with a position resolution of $\sigma_{x y} \sim 0.64 \mathrm{~cm} / \sqrt{E}$.

\subsection{Electronics and Data Rates}

The GlueX experiment requires data rates and volumes that are unprecedented at Jefferson Lab. High data rates and the need for high-speed Level-1 triggering have lead to the development of a number of custom VME electronics boards. These include two different flash ADC modules, one that samples at $250 \mathrm{MHz}$ and another that samples at $125 \mathrm{MHz}$. The $250 \mathrm{MHz}$ version will be used for calorimetry. It will also supply the primary inputs to the $\mathrm{L} 1$ trigger. The modules will be connected through a high 


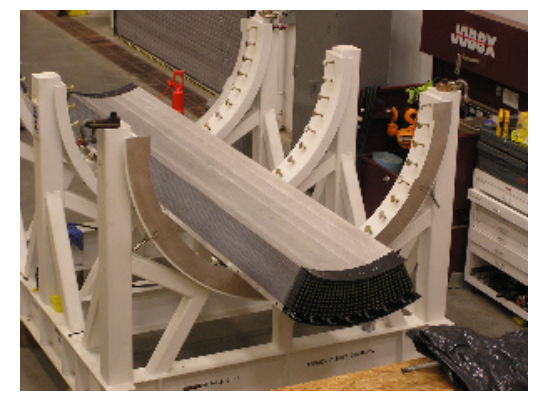

Figure 7. Seven modules from the Barrel Calorimeter (BCAL) installed on the staging support. Each module is made from approximately $17 \mathrm{k}$ scintillating fibers sandwiched between layers of lead[7]. Once all 48 modules are stacked, they will form a cylindrical shape that will then be inserted into the GlueX Solenoid as a whole.

Figure 8. The GlueX Forward Calorimeter (FCAL) installed in Hall-D. The FCAL consists of 2800 lead-glass (type F8-00) blocks stacked in a circular frame. Each block is read out by a PMT powered by a CrockoftWalton base controlled through a CANN system.

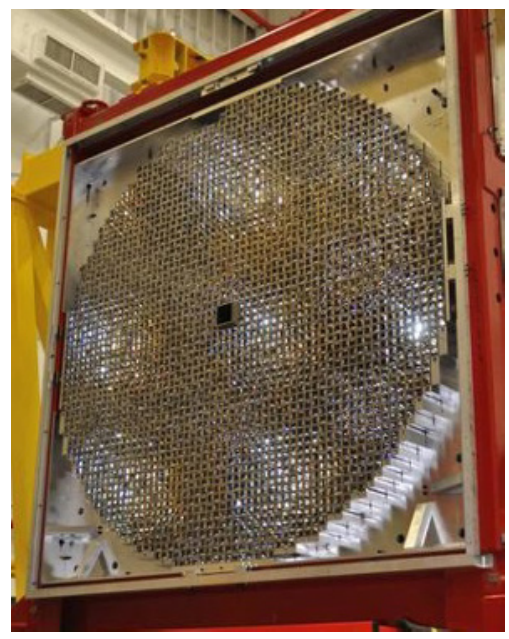

speed serial connection to a central module that will continuously integrate the signals for the crate. Likewise, the sums from each crate will be added together digitally so that a threshold can be applied to total sum of the 2800 element FCAL and the 1536 element BCAL. A L1 trigger rate of $20 \mathrm{kHz}$ is expected for low luminosity running during the first year of data taking. High luminosity running will increase the L1 trigger rate to $200 \mathrm{kHz}$ and require a L3 trigger be implemented. This will use a high speed computer farm to reduce the $3 \mathrm{~GB} / \mathrm{s}$ of data read from the front ends down to $300 \mathrm{MB} / \mathrm{s}$ for archival to tape. GlueX anticipates 1 petabyte of data to tape per year from both low luminosity and high luminosity running.

\section{Schedule and Summary}

The GlueX experiment will search for the lightest exotic hybrid mesons that are currently predicted by LQCD. This will be done using the GlueX detector and its linearly polarized photon source. The fixed target, solenoid geometry will provide a large acceptance while maintaining good resolution. The GlueX detector will produce unprecedented amounts of data for a Jefferson Lab experiment at $1 \mathrm{~PB} / \mathrm{yr}$ at high luminosity running. Commissioning of the upgraded accelerator is expected to begin 
INPC 2013

in Oct. 2013 with commissioning of the GlueX detector beginning one year later. Production data taking for the GlueX experiment is expected to begin in 2015.

\section{Acknowledgements}

This work was supported by DOE Contract No. DE-AC05-06OR23177, under which Jefferson Science Associates, LLC, operates Jefferson Laboratory.

\section{References}

[1] Proposal for JLab Experiment E12-06-102: Mapping the Spectrum of Light Quark Mesons and Gluonic Excitations with Linearly Polarized Photons, http://www.jlab.org/exp_prog/proposals/06/PR12-06-102.pdf (2006)

[2] J.J. Dudek, Phys. Rev. D 84, 074023 (2011)

[3] J.J. Dudek, et al., Phys. Rev. Letters 103, 262001 (2009)

[4] C.A. Meyer, Y. VanHaarlem, Phys. Rev. C 82, 025208 (2010)

[5] C.J. Morningstar, M. Peardon, Phys. Rev. D 60, 034509 (1999)

[6] J.J. Dudek, et al., Phys. Rev. D 79, 094504 (2009)

[7] B. Leverington, et al., NIM A 596, 327 (2008) 
